\title{
Enhanced filopodium formation and stem-like phenotypes in a novel metastatic head and neck cancer cell model
}

\author{
YU-SYUAN CHEN $^{1 *}$, WEI-LI HUANG ${ }^{2 *}$, SHU-HAO CHANG $^{3}$, KUO-WEI CHANG ${ }^{1,2}$, \\ SHOU-YEN KAO ${ }^{2,4}$, JENG-FAN LO $\mathrm{LO}^{1,2,4,5}$ and PEI-FEN SU $\mathrm{SU}^{2,3}$ \\ ${ }^{1}$ Institute of Oral Biology and ${ }^{2}$ Department of Dentistry, School of Dentistry, National Yang-Ming University, Taipei; \\ Departments of ${ }^{3}$ Medical Research and Education and ${ }^{4}$ Stomatology, Oral Maxillofacial Surgery, \\ Taipei Veterans General Hospital, Taipei; ${ }^{5}$ Graduate Institute of Chinese Medical Science and \\ Institute of Medical Science, China Medical University, Taichung, Taiwan, R.O.C.
}

Received July 30, 2013; Accepted September 4, 2013

DOI: $10.3892 /$ or.2013.2772

\begin{abstract}
Head and neck squamous cell carcinoma (HNSCC) is the sixth most common cancer in the world, and metastasis is the major cause of cancer-related mortality. Prevention or elimination of metastasis may improve the survival of cancer patients; however, the availability of systemic HNSCC cell model with which to investigate the mechanisms of metastasis is limited. In the present study, we established a set of metastatic cell lines from HNSCC cells. In combination with their low-tumorigenic and high-tumorigenic ancestor cell lines, a cell model containing cell lines with varying malignant characteristics was established. Transcriptome analysis revealed distinct signatures among the metastatic cell lines, in comparison to the ancestor cell lines. Signaling of GTPase RhoA and mammalian embryonic stem cell pluripotency were identified in the metastatic cells. Moreover, we examined the expression of genes related to epithelial-mesenchymal transition (EMT) (Snail, Slug, Twist, vimentin and fibronectin) and stemness (Oct4, Nanog and Bmi1). The capabilities of the cells for migration, invasion, spheroid formation and pulmonary colonization in nude mice were determined. Together, we demonstrated gain of Slug expression, filopodium formation and stem-like properties in metastatic HNSCC cells, rendering this model a powerful tool for the development of diagnostic biomarkers and identification of therapeutic targets for HNSCC.
\end{abstract}

Correspondence to: Dr Jeng-Fan Lo, Institute of Oral Biology, School of Dentistry, National Yang-Ming University, No. 155, Section 2, Li-Nong Street, Taipei 11217, Taiwan, R.O.C.

E-mail: jflo@ym.edu.tw

Dr Pei-Fen Su, Department of Medical Research and Education, Taipei Veterans General Hospital, No. 201, Section 2, Shih-Pai Road, Taipei 11217, Taiwan, R.O.C.

E-mail: pfsu@vghtpe.gov.tw

*Contributed equally

Key words: metastasis, filopodia, stemness, invasion, pulmonary colony, epithelial-mesenchymal transition

\section{Introduction}

Metastasis is the endpoint of tumorigenesis and is a major cause of cancer-related mortality (1). Prevention or elimination of this level of disseminated cancer progression is essential to reduce morbidity and mortality $(1,2)$. Head and neck squamous cell carcinoma (HNSCC) is the sixth most common cancer in the world (3). Although therapeutic regimens for HNSCC have been improved, the survival rate of HNSCC patients has not markedly increased due to locoregional and/or distant recurrence/metastasis (4). Therefore, to develop effective therapeutic or preventive strategies to overcome the progression of this cancer, elucidation of the mechanisms of metastasis is an urgent issue.

Mounting evidence supports the hypothesis that tumors contain a small subpopulation of cells, called cancer stem cells (CSCs) or cancer initiating cells (CICs), which exhibit self-renewal capacity, and are responsible for tumor growth and metastasis (5). In addition, we previously demonstrated the existence of stemness-possessing cells in $\operatorname{HNSCC}(6,7)$. Metastatic potential depends on multiple factors, including the regulation of cell growth, survival, angiogenesis and invasion. Furthermore, epithelial-mesenchymal transition (EMT) is recognized as a crucial event in the metastatic process (8) and may enhance stemness and tumor-initiating properties (9-11). However, modulators of metastatic HNSCC that participate in the EMT process and stemness remain unclear.

Although tumorigenic and metastatic head and neck carcinoma cell lines are available, the genetic variation among these cell lines makes it difficult to compare the observations derived from these different cell lines. Therefore, developing a systemic metastatic cellular model derived from the same ancestor cellular lineage could be an important tool for investigating metastasis in head and neck cancer. In the present study, we aimed to develop a cellular model consisting of cell lines possessing differential malignant potential but with the same genetic background. Such a system may allow us to avoid the variation found in cell lines derived from different individuals or types of tissues.

Previously, we established the tumorigenic SASVO3 cell line, which possesses enhanced in vivo tumorigenicity, 
from parental HNSCC SAS cells (12). SASVO3 cells exhibit enhanced cancer stem cell properties, including an increase in sphere-forming activity, the number of side population cells and the expression of the stem cell marker Bmil. Herein, we successfully established a metastatic carcinoma cell line from pulmonary metastatic nodules of SASVO3 cells. Using these cell lines, derived from the same parental cancer cell line and exhibiting an increase in malignancy potential, we investigated the underlying mechanisms of tumorigenesis and metastasis by transcriptome analysis and cellular assays.

\section{Materials and methods}

Cell culture. Human HNSCC SAS cells and the various derived cell lines, including SASVO3, SASVO3M-1 and SASVO3M-5, were cultured in Dulbecco's modified Eagle's medium (DMEM) (Gibco-BRL). The medium was supplemented with $10 \%$ fetal bovine serum (FBS) (HyClone Laboratory), $2 \mathrm{mM}$ L-glutamine, $100 \mathrm{U} / \mathrm{ml}$ penicillin and $100 \mu \mathrm{g} / \mathrm{ml}$ streptomycin sulfate. The cells were maintained at $37^{\circ} \mathrm{C}$ in a humidified atmosphere containing $5 \% \mathrm{CO}_{2}$.

Establishment of the metastatic cell lines. Female nude mice, aged 6-8 weeks, were obtained from the National Laboratory Animal Breeding and Research Center, Taipei, Taiwan. All mice were maintained under standard pathogen-free laboratory conditions. Animal experiments were conducted according to the institutional guidelines established by the Animal Core Facility and IACUC of the Veterans General Hospital, Taipei, Taiwan. SASVO3 cells at a density of $5 \times 10^{5}$ cells $/ 100 \mu 1$ PBS were injected into nude mice via the tail vein. The mice were euthanized 65 days after injection, and metastatic nodules were removed from the lungs. Lung tissues were subjected to pathological examination and in vitro cell culture studies. For histological analyses, lung tissues were fixed with buffered-formalin and embedded in paraffin. Sections were stained using standard hematoxylin and eosin protocol. For cell line generation, metastatic nodules were torn into tiny pieces and grown in medium consisting of DMEM and defined keratinocyte serum-free medium (KSFM) (Gibco-BRL) at a ratio of 1:2. The outgrowth epithelial tumor cells were cloned and adapted to complete DMEM. Two clones derived from the metastatic nodules were designated as SASVO3M-1 and SASVO3M-5. The abbreviations for SASVO3, SASVO3M-1 and SASVO3M-5 cells are VO3, M-1 and M-5, respectively.

Global transcription and pathway analyses. Total RNA was extracted from the parental SAS cells, as well as the derived cell lines, using TRIzol reagent (Invitrogen Life Technologies). RNA purity was determined based on the OD260/280 ratio and electrophoretic analysis. Genome-wide gene expression profiling was performed using the human HT-12 v4 BeadChip array (Illumina, Inc.). This system utilizes $>47,000$ probes, which cover 29,000 transcripts. Data were extracted from GenomeStudio software (Illumina, Inc.). After quantile normalization, a total of 47,320 probes were included for analysis. The biological functions and pathways of the selected genes were interpreted by Ingenuity pathway analysis (Ingenuity ${ }^{\circledR}$ Systems, Inc.)
Focus formation assay. Parental SAS cells and the derived cell lines were plated $\left(1.8 \times 10^{6}\right.$ cells) onto $60-\mathrm{mm}$ dishes and grown in DMEM containing 2\% FBS for 6 days. Cells were subsequently fixed with methanol and stained with $10 \%$ Giemsa solution in PBS.

Fluorescence-activated cell sorting (FACS). Primary antibodies against integrin $\alpha v \beta 6$ (Millipore), uPAR (American Diagnostica, Inc.) and CD133 (Miltenyi Biotec) were used to determine the expression of proteins on the cell surface. APC goat anti-mouse (Ig) (Becton-Dickinson and Company) was used as a secondary antibody. The isotype antibody was purified mouse IgG1 (BioLegend). Primary and secondary antibodies were incubated serially at $4^{\circ} \mathrm{C}$ for $30 \mathrm{~min}$. Analyses were performed using a FACScanto flow cytometer (BD Biosciences).

Immunoblotting. The antibodies used in this study included Snail (3895; Cell Signaling Technology, Inc.), Slug (ab27568; Abcam), Twist (sc-15393; Santa Cruz Biotechnology, Inc.), vimentin (550513) and fibronectin (610078; both from BD Biosciences), GAPDH (MAB374; Millipore), FAK (sc557), Tyr 925-phosphorylated FAK (sc-11766) and Oct4 (sc-9081) (all from Santa Cruz Biotechnology, Inc.), Nanog (ab21624; Abcam) and Bmil (2830; Cell Signaling Technology, Inc.). For immunoblotting, the cell pellets were homogenized in $200 \mathrm{ml}$ lysis buffer (50 mM HEPES, pH 7.5, $150 \mathrm{mM} \mathrm{NaCl}$, $10 \%$ glycerol, $1.5 \mathrm{mM} \mathrm{MgCl}_{2}, 1 \%$ Triton X-100) and incubated on ice for $20 \mathrm{~min}$. After centrifugation at $13,000 \mathrm{x} \mathrm{g}$ for $30 \mathrm{~min}$ at $4^{\circ} \mathrm{C}$, the supernatant containing the protein extracts was collected. Protein concentrations were determined using the Bio-Rad protein assay. Immunoblot analyses were performed as described previously (12).

Immunofluorescence staining and confocal microscopy. Parental SAS cells and the derived cell lines were plated on glass coverslips in 6-well plates. For E-cadherin immunofluorescence staining, cells were fixed with $4 \%$ paraformaldehyde/phosphate-buffered saline (PBS) and permeabilized with $0.5 \%(\mathrm{v} / \mathrm{v})$ Triton X-100 in PBS, followed by incubation with blocking solution [1\% BSA and $0.2 \%(\mathrm{v} / \mathrm{v})$ Tween-20 in PBS]. Cells were incubated with primary antibodies against E-cadherin (BD Transduction Laboratories), and subsequently incubated with the secondary antibody conjugated with FITC (Sigma-Aldrich). Nuclei were counterstained with DAPI, and the cells were mounted using fluorescence mounting medium (Dako). Fluorescence images were captured using a Zeiss Axio Observer A1 microscope. For F-actin staining, cells were fixed with $4 \%$ paraformaldehyde, incubated in blocking solution and stained with TRITC-conjugated phalloidin (Sigma-Aldrich). The cells were subsequently counterstained with DAPI and examined using an Olympus FV1000 confocal microscope.

Cell migration assay. Cell migration assays were performed using an Oris $^{\mathrm{TM}}$ cell migration assembly kit (Platypus Technologies) according to the manufacturer's instructions. In brief, the assay utilizes Oris ${ }^{\mathrm{TM}}$ cell seeding stoppers to restrict cell seeding to the outer annular region of the wells. SAS cells and the derived cell lines were plated onto each well at the 
A

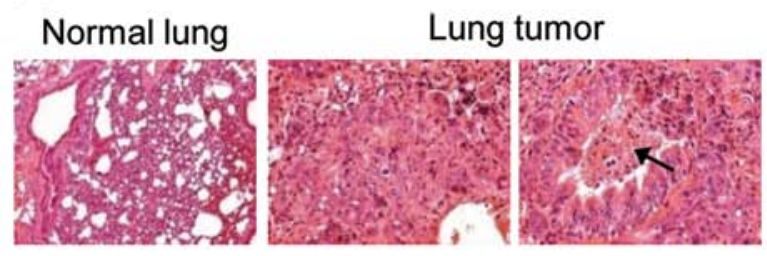

B

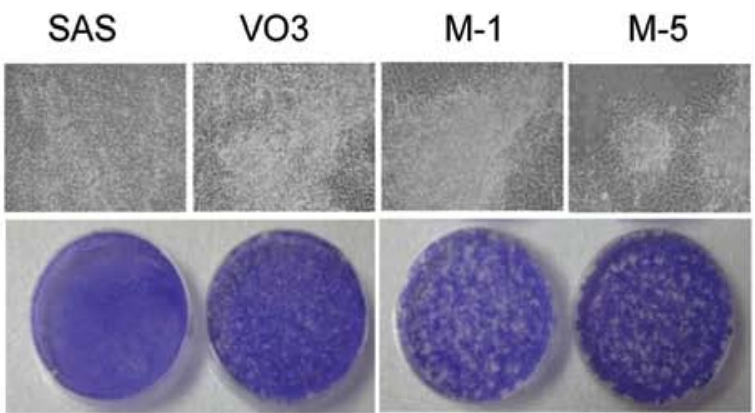

C

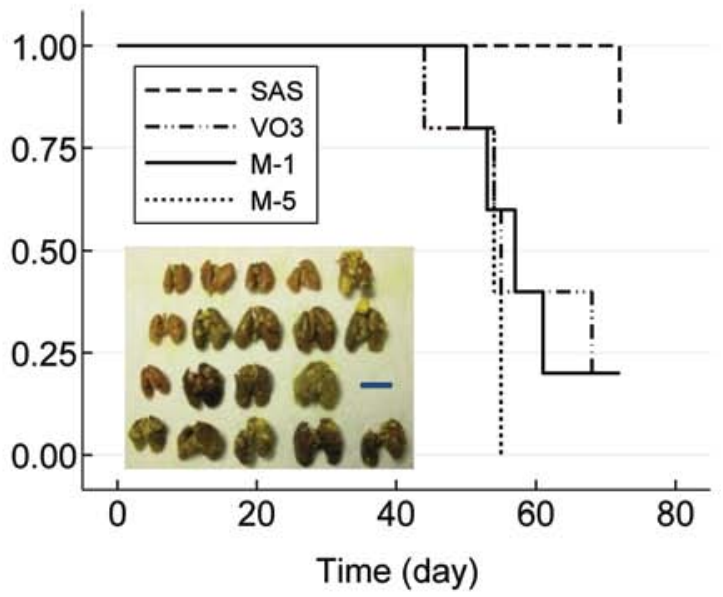

Figure 1. Establishment and characterization of the metastatic HNSCC cell lines. (A) Hematoxylin and eosin staining of murine lung tissue and metastatic tumors. The arrow indicates tumor cells that had invaded into the trachea. (B) Focus formation assay. Upper panels, microphotographs; lower panels, Giemsa stain. (C) Kaplan-Meier survival analysis of nude mice injected with SAS and the derived cell lines: SAS or VO3 cells (5x10 5 cells); M-1 or M-5 cells $\left(5 \times 10^{4}\right.$ cells). Image shows metastatic lung colonies fixed in Bouin's solution; scale bar, $10 \mathrm{~mm}$. HNSCC, head and neck squamous cell carcinoma.

indicated density in serum-free DMEM and allowed to attach for $6 \mathrm{~h}$ at $37^{\circ} \mathrm{C}$. The stopper was subsequently removed to form an unseeded region at the center of each well. An image of the clear detection zone was captured as a control for cell migration. The cells were then incubated at $37^{\circ} \mathrm{C}$ for $16 \mathrm{~h}$ to permit cell migration into the detection zone. The cells were stained with Calcein AM fluorescent dye (Sigma-Aldrich) for $1 \mathrm{~h}$ before images were captured using a Zeiss Axio Observer A1 microscope.

Cell invasion assay. Cell invasion assays were performed using an Oris ${ }^{\mathrm{TM}}$ 96-well cell migration assay kit following the manufacturer's instructions. In brief, a thin layer of basal membrane was coated by rinsing each well with $3.5 \mathrm{mg} / \mathrm{ml}$ BME coating solution, after which the seeding stopper was pressed down into each well. The cells were starved in medium containing $1 \%$ FBS for $18 \mathrm{~h}$ prior to plating. The cells were incubated for $6 \mathrm{~h}$ to permit cell attachment. The stopper was then removed, and $40 \mu \mathrm{l}$ BME coating solution was added to create a 3-D BME layer. After incubating for $48 \mathrm{~h}$, the cells were stained for the cell migration assay.

Sphere formation assay. SAS cells and the derived cell lines were cultured in tumor sphere medium consisting of serum-free DMEM/F12 medium, N2 supplement (both from Gibco-BRL), $10 \mathrm{ng} / \mathrm{ml}$ human recombinant basic fibroblast growth factor-basic (FGF) and $10 \mathrm{ng} / \mathrm{ml}$ epidermal growth factor (EGF) (R\&D Systems). Cells were plated at a density of $7.5 \times 10^{4}$ to $1 \times 10^{5}$ live cells/100-mm dish, and the medium was replaced every other day until tumor sphere formation was observed ( 4 weeks) (7).

In vivo pulmonary metastasis assay. Nude mice were randomly grouped into sets of 5 mice each. Cells at the indicated cell number were individually injected into mice via the tail vein. The date of animal death or euthanasia was recorded, and the lung tissue from these mice was fixed with Bouin's solution.

Statistical analyses. Kaplan-Meier survival was analyzed with the Statistical Package for the Social Sciences (SPSS) statistical program version 17.0 (SPSS, Inc.).

\section{Results}

Establishment of the metastatic HNSCC cell lines. To establish a cellular model for investigating the progression of metastasis, we attempted to generate metastatic cell lines from the highly tumorigenic SASVO3 (VO3) cell line, which was previously developed from parental SAS cells (12). Empirically, we induced pulmonary metastatic tumors by injecting VO3 cells into the tail vein of nude mice. Pathological analysis revealed that the tumor cells subsequently found in the lung were capable of invading into the trachea (Fig. 1A). Two metastatic cell line clones were generated and designated as M-1 and M-5. The in vitro malignancy of VO3, M-1 and M5 were further examined by a focus formation assay. As shown in Fig. 1B, these metastatic cell lines exhibited significantly enhanced focus formation when compared with the original VO3 cells, even when the cells were not confluent. In contrast, the SAS cells with reduced tumorigenicity rarely formed foci, even at full confluence (Fig. 1B).

Enhanced pulmonary metastatic activity of metastatic HNSCC cells. To compare the lung colonization activity of cell lines with different malignant potential, SAS cells and cells from the 3 derived cell lines were collected and injected into nude mice via the tail vein. The results showed that the metastatic M-1 and M-5 cells, even when injected at a 10-fold 
Heatmap

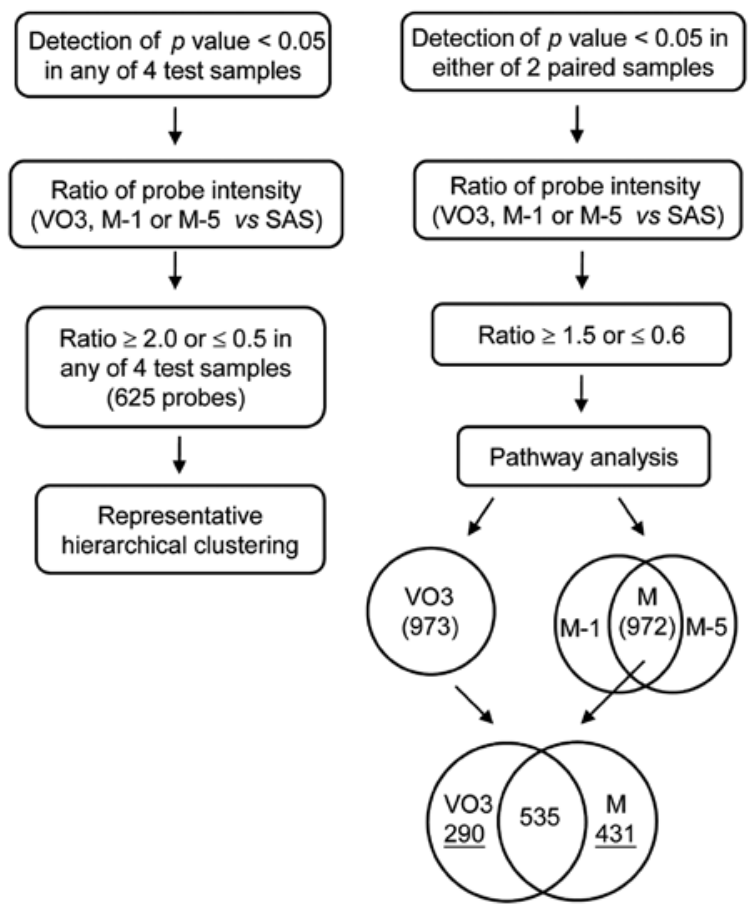

Figure 2. Flow chart of the selection algorithm. Numbers indicate the number of genes in each group. The genes identified as common to both metastatic cell line clones are designated as 'M.'

lower cell number than SAS or VO3 cells, were capable of inducing more pulmonary metastatic colonies and shortening the overall survival of the injected mice (Fig. 1C). In contrast, non-metastatic SAS cells rarely formed pulmonary metastatic colonies. The data thus revealed that the newly established metastatic cells possessed an enhanced ability to extravasate and proliferate in the lung parenchyma as compared to the ancestor VO3 cells.

Distinct transcriptome signatures of the metastatic HNSCC cells. To understand the transcriptional changes in the cell lines with different malignant potential, genome-wide gene expression profiling of SAS, VO3, M-1 and M-5 cells was carried out. Scatter plot analysis revealed a high correlation of metastatic M-1 and M-5 clones $\left(\mathrm{R}^{2}=0.9941\right)$. After quantile normalization, 47,320 probes were subjected to 2 systemic analyses to measure the correlation of cells with differing malignant potential (heatmap) and to select the genes that displayed a marked alteration in parallel with malignancy (fold-change). A schematic of the transcriptome data analysis is shown in Fig. 2. The criteria of selection were: i) to limit false results arising from low-expression genes. Probes with detection of a p-value $\geq 0.05$ in any of the 4 test samples (heatmap), or in either of the paired samples (fold-change) were excluded. ii) The ratio of probe intensity of VO3, M-1 or M-5 vs. that of SAS was calculated. iii) Cutoff values of the ratio were as indicated in Fig. 2.

As shown in Fig. 3, hierarchical clustering revealed a differential signature in cells with varying malignant potential. The signatures of the 2 metastatic cell lines were the most closely related to each other, but were most distinct from the

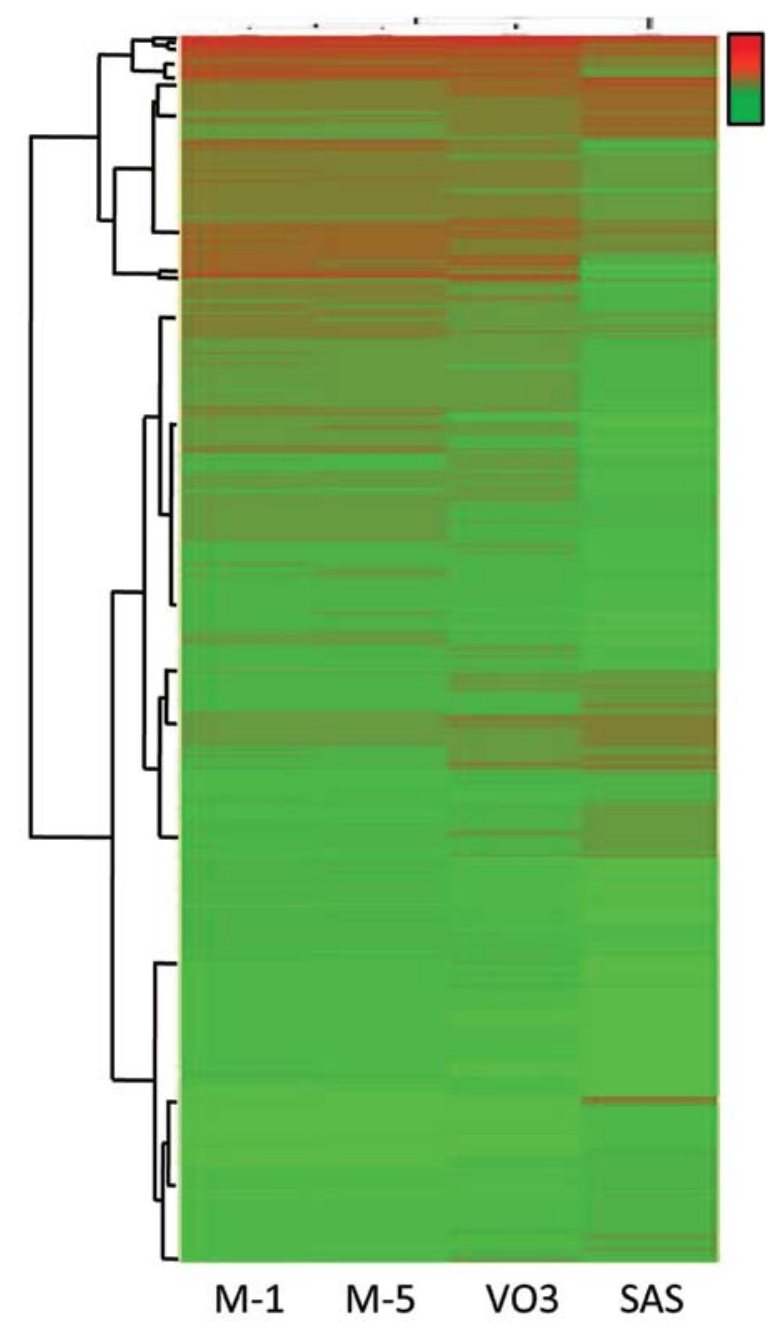

Figure 3. Representative hierarchical clustering of SAS and the derived cell lines. The x-axis represents the correlation of parental SAS, VO3, M-1 and M-5 cells. The y-axis represents the clustering of 625 selected genes. Log intensity ranging from 4.5 to 1.5 is depicted as red to green.

non-metastatic SAS cells. However, the signature of highly tumorigenic VO3 cells was in-between the aforementioned cell lines and contained certain uniquely expressed genes. To transcriptionally profile these changes between the highly tumorigenic cells and metastatic cells, genes with a statistically significant difference in expression in the VO3, M-1 and M-5 cells were selected and subjected to pathway analysis. There were 825 and 972 ingenuity pathway analysis (IPA)-identified genes displaying significant changes in VO3 cells and with common preservation in M-1 and M-5 cells (designate M), respectively. Furthermore, a total of 535 overlapping genes were observed in both tumorigenic and metastatic cells, while 209 and 431 genes were uniquely found in VO3 cells and commonly reserved in $\mathrm{M}$ cells, respectively (Fig. 2, right). Biological pathways involved in VO3 (825) and common M (972) gene sets were then analyzed. Several canonical pathways were identified in both the VO3 and the common $M$ groups, including the integrin signaling pathway, VDR/ RXR activation pathway and IL-17A, which has a role in psoriasis. Of note, Notch signaling was found exclusively in the highly tumorigenic cells, while interferon signaling, RhoA signaling and Oct4, which plays a role in mammalian embry- 
Table I. Ingenuity pathway analysis of top canonical pathways in the highly tumorigenic or metastatic cell lines.

\begin{tabular}{|c|c|c|}
\hline Pathway name & P-value & Ratio \\
\hline \multicolumn{3}{|l|}{ Highly tumorigenic cells (VO3) } \\
\hline VDR/RXR activation ${ }^{\mathrm{a}}$ & $1.65 \mathrm{E}-05$ & $13 / 81(0.160)$ \\
\hline Integrin signaling & 4.17E-04 & $19 / 207(0.092)$ \\
\hline Notch signaling & 7.5E-04 & $7 / 43(0.163)$ \\
\hline Cyclins and cell cycle regulation & $1.33 \mathrm{E}-03$ & $10 / 89(0.112)$ \\
\hline Role of IL-17A in psoriasis & $1.45 \mathrm{E}-03$ & $4 / 13(0.308)$ \\
\hline ILK signaling ${ }^{\mathrm{b}}$ & $1.67 \mathrm{E}-03$ & $17 / 192(0.089)$ \\
\hline Role of IL-17F in allergic inflammatory airway diseases & $2.11 \mathrm{E}-03$ & $7 / 48(0.146)$ \\
\hline Interferon signaling & $2.25 \mathrm{E}-03$ & $6 / 36(0.167)$ \\
\hline Glioma invasiveness signaling & 2.29E-03 & $8 / 60(0.133)$ \\
\hline Neurotrophin/TRK signaling & $2.5 \mathrm{E}-03$ & $9 / 75(0.120)$ \\
\hline \multicolumn{3}{|l|}{ Metastatic cells (M-1 and M-5) } \\
\hline Interferon signaling & $6.39 \mathrm{E}-06$ & $10 / 36(0.278)$ \\
\hline Role of IL-17A in psoriasis & 2.61E-05 & $6 / 13(0.462)$ \\
\hline VDR/RXR activation ${ }^{\mathrm{a}}$ & 4.95E-05 & $14 / 81(0.173)$ \\
\hline RhoA signaling & 2.24E-04 & $16 / 114(0.140)$ \\
\hline Antigen presentation pathway & $5.63 \mathrm{E}-04$ & $8 / 40(0.20)$ \\
\hline Role of tissue factor in cancer & $5.78 \mathrm{E}-04$ & $15 / 114(0.132)$ \\
\hline Integrin signaling & $6.61 \mathrm{E}-04$ & $22 / 207(0.106)$ \\
\hline Clathrin-mediated endocytosis signaling & $1.8 \mathrm{E}-03$ & $20 / 196(0.102)$ \\
\hline Role of Oct 4 in mammalian embryonic stem cell pluripotency & $2.17 \mathrm{E}-03$ & $8 / 45(0.178)$ \\
\hline Virus entry via endocytic pathways & 2.37E-03 & $12 / 99(0.121)$ \\
\hline
\end{tabular}

${ }^{\mathrm{a}} \mathrm{VDR}$, vitamin D receptor; RXR, retinoic acid receptor; ${ }^{\mathrm{b}} \mathrm{ILK}$, integrin linked kinase.

onic stem cell pluripotency, were significantly altered in the metastatic cells. Table I depicts the top 10 canonical pathways identified by IPA. Table II shows the top 5 upregulated and downregulated genes uniquely identified in the VO3 or metastatic cells. Collectively, the transcriptome analysis suggests that metastatic HNSCC cells may have profound changes associated with cell movement and stemness activity.

Acquirement of EMT in tumorigenic cells and increased expression of focal adhesion-related genes and increased filopodium formation in metastatic HNSCC cells. To verify the differential expression of cell movement-related genes identified from transcriptome analysis, the protein levels in the distinct cell lines of the above-mentioned genes were determined. Highly tumorigenic VO3 cells displayed upregulation of vimentin and fibronectin (Fig. 4A) and downregulation of the epithelial marker E-cadherin (Fig. 4C-a), suggesting that a gain of EMT may have occurred in VO3 cells. In the 2 metastatic clones, the expression patterns of the 3 genes listed above remained similar, without any significant change. Moreover, the protein levels of genes related to the EMT transcription factor Slug, focal adhesion $\left(\alpha_{v} \beta_{6}\right.$ and p-FAK), and proteolytic degradation, urokinase-type plasminogen activator receptor (uPAR) were increased in the metastatic cells (Fig. 4A and B). However, no obvious changes in Snail or Twist proteins (EMT-related transcription factors) in the 4 tested cell lines were observed (Fig. 4A).
IPA analysis identified the upregulation of small GTPase RhoA signaling in the metastatic cell lines (Table I). To further verify downstream activation of RhoA signaling, the polymerization of F-actin was characterized using phalloidin staining. The metastatic M-5 clone displayed significant actin reorganization in lamellipodia, as well as filopodium formation, when compared to the parental SAS cells (Fig. 4C-b). The highly tumorigenic VO3 cells also showed augmentation of lamellipodia, but less of filopodia (Fig. 4C-b). The mobility assays revealed that the migratory ability of VO3 and M-5 cells was higher than that of the parental SAS cells (Fig. 4C-c). Additionally, the invasive ability of M-5 cells was moderately higher than that of VO3 cells (Fig. 4C-d). Taken together, our data revealed that actin-polymerization, cell migration activity and partial EMT changes occurred in the highly tumorigenic VO3 cells, while focal adhesion, filopodium formation and invasive activity were enhanced in the metastatic cells.

Increased stemness-associated characteristics in metastatic HNSCC cells. Transcriptome analysis revealed activation of the canonical Oct4 pathway, which plays a role in mammalian embryonic stem cell pluripotency, in the metastatic M-1 and M-5 cells (Table I). Hence, we examined the acquisition of stem cell properties in the metastatic cells by sphere formation which is one of the characteristics of stemness (7). The metastatic cells showed increased sphere formation ability in 
A

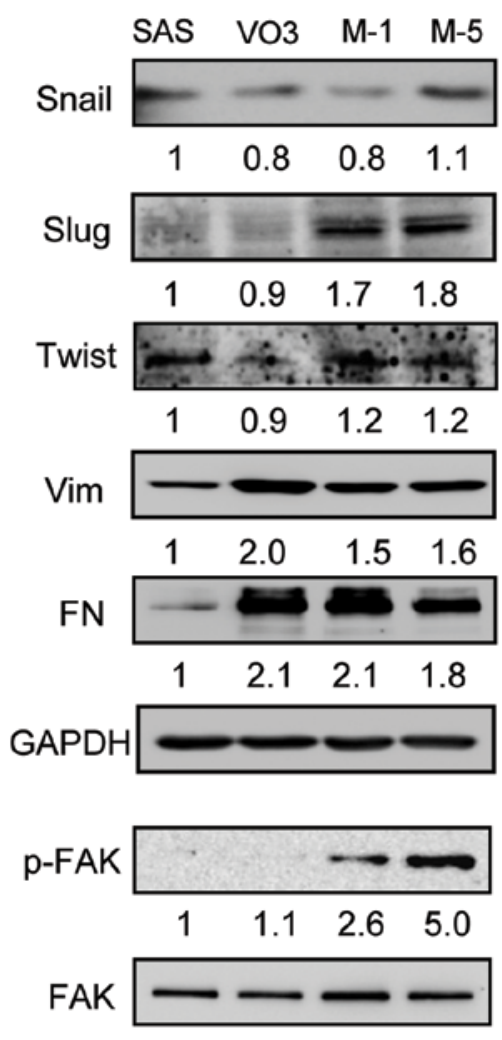

B

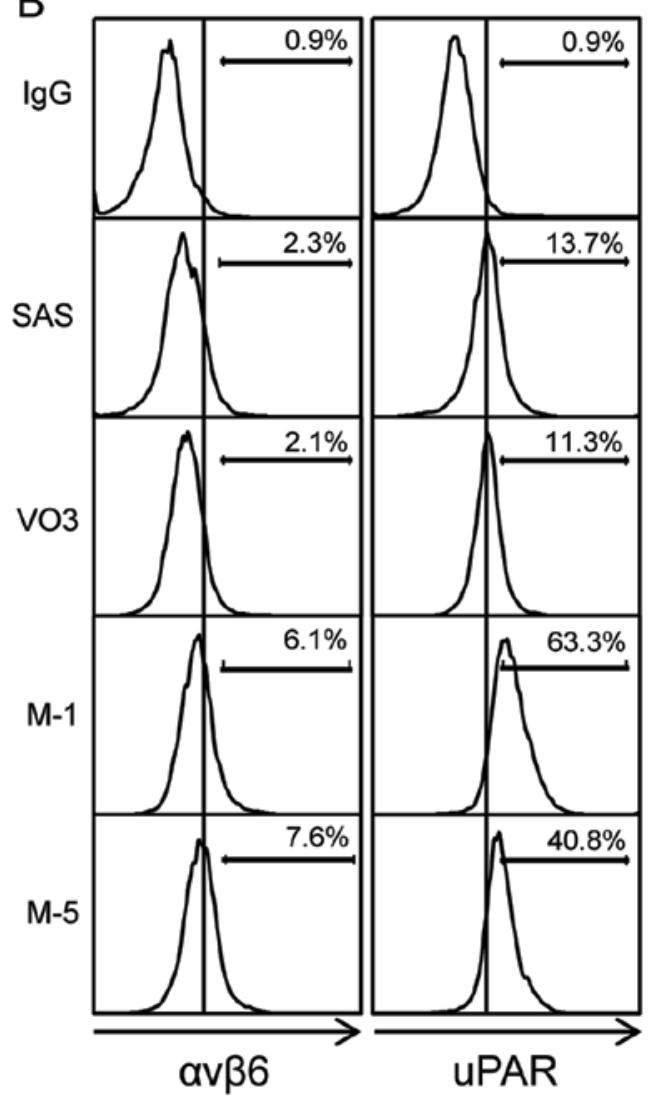

C

a

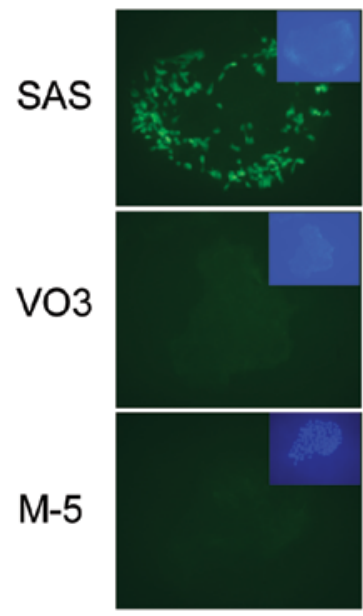

b

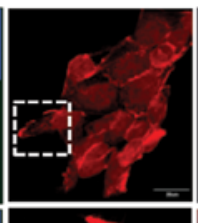

C

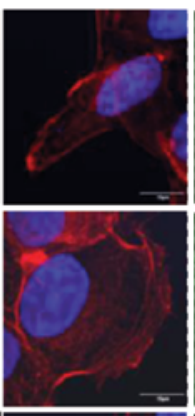

d

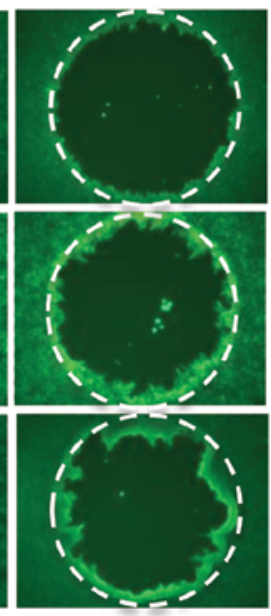

Figure 4. Analysis of mobility-related genes and functional assay of SAS and the derived cell lines. (A) Immunoblot analysis of EMT- and focal adhesionassociated proteins. Vim, vimentin; FN, fibronectin. (B) FACS analysis of integrin $\alpha v \beta 6$ and uPAR. (C) (a) Immunofluorescence analysis of E-cadherin. (b) Phalloidin staining of F-actin. (c) Cell migration activity examined by Oris cell migration assembly kit. (d) Cell invasion activity examined by Oris cell invasion assay in the presence of basement membrane extract. SAS, poorly tumorigenic cells; VO3, highly tumorigenic cells and M-5, metastatic cells. Arrow indicates filopodia. EMT, epithelial-mesenchymal transition; uPAR, urokinase-type plasminogen activator receptor.

regards to both sphere size and number as compared to the parental SAS cells that rarely form spheres and VO3 cells that displayed moderate sphere formation ability (Fig. 5A and B). Furthermore, the expression of the stem cell markers, CD133, Oct4 and Bmil, was also augmented in the metastatic cells, along with a moderate increase in Nanog protein levels (Fig. 5C and D).

\section{Discussion}

Metastasis is an end stage of cancer and is the major cause of cancer-related death. Investigation into the mechanisms underlying metastasis may lead to improvements in diagnosis and therapy. Our previous study generated the highly tumorigenic cell line SASVO3, which acquired increased stem-like proper- 
Table II. Ingenuity pathway analysis of the most significant 5 genes uniquely altered in the highly tumorigenic or metastatic cells.

Gene symbol (name)

Log ratio

Highly tumorigenic cells

Upregulated genes

BGN (biglycan)

HEG1 (HEG homolog 1)

SERPINB3 [serpin peptidase inhibitor, clade B (ovalbumin), member 3]

SERPINE1 [serpin peptidase inhibitor, clade E (nexin, plasminogen activator inhibitor type 1), member 1]

EFEMP1 (EGF containing fibulin-like extracellular matrix protein 1)

Downregulated genes

HCLS1 (hematopoietic cell-specific Lyn substrate 1)

UST (uronyl-2-sulfotransferase)

H2AFY2 (H2A histone family, member Y2)

CADM4 (cell adhesion molecule 4)

ENO2 (enolase 2)

Metastatic cells

Upregulated genes

GJB2 (gap junction protein, $\beta$ )

PPP1R14C [protein phosphatase 1, regulatory (inhibitor) subunit 14C]

S100A7 (S100 calcium binding protein A7)

SAA1 (serum amyloid A1)

SGPP2 (sphingosine-1-phosphate phosphatase 2)

Downregulated genes

MT1G (metallothionein 1G)

${ }^{\mathrm{a}}$ For the highly tumorigenic cells, the ratio is the intensity of VO3 vs. SAS cells. For metastatic cells, the ratio is the intensity of M5 vs. SAS cells.

ties in comparison to the parental SAS cell line (12). Here, we showed that EMT-related genes (vimentin, fibronectin and E-cadherin) and cell migration activity also increased in the highly tumorigenic cells. Moreover, we demonstrated that the derived metastatic cell lines (SASVO3-M-1 and SASVO3-M-5) acquired further alterations in canonical pathways, such as small GTPase signaling and Oct4-mediated pluripotency signaling. Expression of genes involved in focal adhesion (integrin $\alpha_{v} \beta_{6}$ and p-FAK), EMT (Slug), ECM degradation (UPAR) and stemness (Oct4, Bmil and CD133) was also augmented. The metastatic cells also displayed significant filopodium formation and increased cell invasion, sphere formation and pulmonary metastatic colony formation ability compared to the SASVO3 cells. Together, our findings suggest that in the current system, partial EMT changes may occur during tumor formation, while further changes in cell invasiveness and survival may be obtained in parallel with the acquisition of metastatic properties.

Lamellipodia and filopodia play pivotal roles in tumor metastasis and invasion (13) and are known to be controlled by small GTPases. The small GTPase family has been reported to be involved in multiple aspects of cancer development including cell proliferation, cell polarity, adhesion, migration and invasion $(14,15)$. Small Rho GTPases act as molecular switches to translate extracellular signals into intracellular events that induce changes in actin organization (16). Rho, Rac and $\mathrm{Cdc} 42$ are the most well-characterized family members and contribute to the formation of stress fibers, lamellipodia and filopodia, respectively (17). Among them, Cdc42 is specifically involved in the generation of cell polarity and in the orientation of migrating cells $(18,19)$. Integrins are cell surface adhesion receptors mediating adhesion stabilization of filopodia (20). The integrin-FAK axis has been shown to direct the proliferation of disseminated metastatic cells (21). A recent study (22) demonstrated that when using extracellular matrix culture conditions, the formation of filopodium-like protrusions is a prerequisite for integrin-adhesion plaque formation, which then triggers FAK/ERK signaling and ultimately permits cell proliferation and the establishment of metastatic colonies. This finding shows that integrin, FAK activation and F-actin reorganization by small GTPases modulate the dissemination and proliferation of metastatic cells. Our 

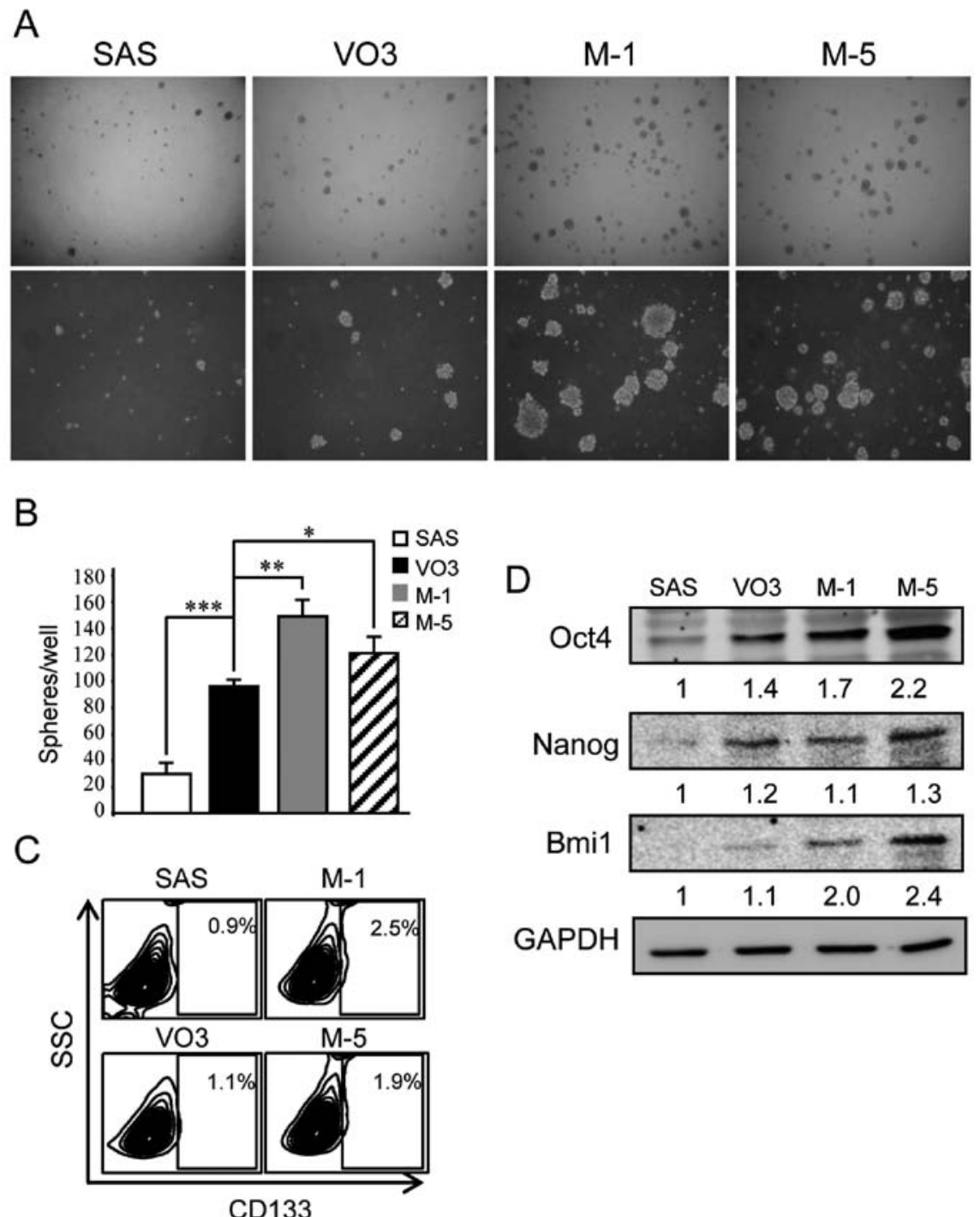

CD133

Figure 5. Determination of stemness-associated activity and stem cell markers. (A) Representative images from the sphere formation assays. (B) Quantification of the sphere numbers. Values are the means $\pm \mathrm{SD}$ of triplicate samples from 3 independent assays. ${ }^{*} \mathrm{p}<0.05 ;{ }^{* *} \mathrm{p}<0.01 ;{ }^{* * *} \mathrm{p}<0.001$. (C) FACS analysis of the stem cell marker CD133. (D) Immunoblot analysis of the stem cell markers Oct4, Nanog and Bmil.

present findings agree with these studies and suggest that our established cellular system may be a useful model for investigating the mechanisms underlying cellular dissemination, cell polarity and cell survival in tumor metastasis.

Slug is one of the transcription factors of EMT and has been shown to regulate cell invasion and metastasis (23). However, in concert with Sox9, these transcription factors can convert differentiated murine luminal cells into mammary stem cells (24). These studies demonstrate that the EMT transcription factor slug may play a role in stem cell maintenance, which supports our finding that the metastatic HNSCC clones possessed both pulmonary metastatic and stem-like activity.

Multiple lines of evidence reveal that Oct4 plays fundamental roles in stem cell self-renewal and pluripotency, as well as somatic cell reprogramming of murine embryonic stem (ES) cells by connecting epigenetic pathways $(25,26)$. In conjunction with Cdk1, Oct4 maintains stemness by inhibiting cellular differentiation (27). In human ES cells, Oct4, Nanog and SOX2 play distinct roles in lineage specification (28). In combination with Bmil, a member of polycomb repressor complex 1
(PRC1), Oct4 is sufficient to reprogram mouse embryonic and adult fibroblasts into induced pluripotent stem (iPS) cells (29). Our pathway analysis demonstrated that Oct4-mediated stem cell pluripotency signaling was significantly altered in the metastatic cells and that Oct 4 and Bmi1 protein levels were increased in metastatic cells. Therefore, the roles of Oct 4 and Bmil in stemness and differentiation, or even metastasis, may be explored using our established metastatic cells.

Overall, our findings suggest that the gain of EMT properties, migratory ability, invasiveness and stem cell-like properties are the major signatures of metastatic cells, along with enhanced malignancy. Furthermore, we established an important cellular system for the future study of metastasis, which may benefit the future treatment of head and neck cancer.

\section{Acknowledgements}

This study was supported by grants from the National Science Council (NSC 99-2314-B-075-041-MY3 and 
NSC 101-2320-B-010-050), the Taipei Veterans General Hospital (V99ER2-004 and V102E2-003) and the Ministry of Education, Aim for the Top University Plan, National Yang-Ming University (101AC-T513).

\section{References}

1. Valastyan S and Weinberg R: Tumor metastasis: molecular insights and evolving paradigms. Cell 147: 275-292, 2011.

2. Eccles SA and Welch DR: Metastasis: recent discoveries and novel treatment strategies. Lancet 369: 1742-1757, 2007.

3. Jemal A, Siegel R, Ward E, Murray T, Xu J and Thun MJ: Cancer statistics, 2007. CA Cancer J Clin 57: 43-66, 2007.

4. Hinerman RW, Mendenhall WM, Morris CG, Amdur RJ, Werning JW and Villaret DB: Postoperative irradiation for squamous cell carcinoma of the oral cavity: 35-year experience. Head Neck 26: 984-994, 2004.

5. Visvader JE and Lindeman GJ: Cancer stem cells in solid tumours: accumulating evidence and unresolved questions. Nat Rev Cancer 8: 755-768, 2008.

6. Wu MJ, Jan CI, Tsay YG, et al: Elimination of head and neck cancer initiating cells through targeting glucose regulated protein 78 signaling. Mol Cancer 9: 283, 2010.

7. Chiou SH, Yu CC, Huang CY, et al: Positive correlations of Oct- 4 and Nanog in oral cancer stem-like cells and high-grade oral squamous cell carcinoma. Clin Cancer Res 14: 4085-4095, 2008.

8. Loboda A, Nebozhyn M, Watters J, et al: EMT is the dominant program in human colon cancer. BMC Med Genomics 4: 9, 2011.

9. Mani SA, Guo W, Liao MJ, et al: The epithelial-mesenchymal transition generates cells with properties of stem cells. Cell 133 704-715, 2008

10. Chen C, Wei Y, Hummel M, et al: Evidence for epithelial-mesenchymal transition in cancer stem cells of head and neck squamous cell carcinoma. PLoS One 6: e16466, 2011.

11. Lo JF, Yu CC, Chiou SH, et al: The epithelial-mesenchymal transition mediator S100A4 maintains cancer-initiating cells in head and neck cancers. Cancer Res 71: 1912-1923, 2011.

12. Chen CY, Chiou SH, Huang CY, et al: Distinct population of highly malignant cells in a head and neck squamous cell carcinoma cell line established by xenograft model. J Biomed Sci 16: 100, 2009

13. Machesky LM: Lamellipodia and filopodia in metastasis and invasion. FEBS Lett 582: 2102-2111, 2008.
14. Leve F and Morgado-Díaz JA: Rho GTPase signaling in the development of colorectal cancer. J Cell Biochem 113: 2549-2559, 2012.

15. Khalil BD and El-Sibai M: Rho GTPases in primary brain tumor malignancy and invasion. J Neurooncol 108: 333-339, 2012.

16. Hall A: Rho GTPases and the actin cytoskeleton. Science 279: 509-514, 1998

17. Etienne-Manneville S and Hall A: Rho GTPases in cell biology. Nature 420: 629-635, 2002

18. Etienne-Manneville S: Polarity proteins in migration and invasion. Oncogene 27: 6970-6980, 2008.

19. Gupton SL and Gertler FB: Filopodia: the fingers that do the walking. Sci STKE 2007: re5, 2007.

20. Arjonen A, Kaukonen R and Ivaska J: Filopodia and adhesion in cancer cell motility. Cell Adh Migr 5: 421-430, 2011.

21. Shibue $T$ and Weinberg RA: Integrin $\beta_{1}$-focal adhesion kinase signaling directs the proliferation of metastatic cancer cells disseminated in the lungs. Proc Natl Acad Sci USA 106: 10290-10295, 2009.

22. Shibue T, Brooks MW, Inan MF, Reinhardt F and Weinberg RA: The outgrowth of micrometastases is enabled by the formation of filopodium-like protrusions. Cancer Discov 2: 706-721, 2012.

23. Liang YJ, Wang QY, Zhou CX, et al: MiR-124 targets Slug to regulate epithelial-mesenchymal transition and metastasis of breast cancer. Carcinogenesis 34: 713-722, 2013.

24. Guo W, Keckesova Z, Donaher JL, et al: Slug and Sox9 cooperatively determine the mammary stem cell state. Cell 148: 1015-1028, 2012

25. Pardo M, Lang B, Yu L, et al: An expanded Oct4 interaction network: implications for stem cell biology, development, and disease. Cell Stem Cell 6: 382-395, 2010.

26. Ding J, Xu H, Faiola F, Ma'ayan A and Wang J: Oct4 links multiple epigenetic pathways to the pluripotency network. Cell Res 22: 155-167, 2012

27. Li L, Wang J, Hou J, et al: Cdk1 interplays with Oct4 to repress differentiation of embryonic stem cells into trophectoderm. FEBS Lett 586: 4100-4107, 2012.

28. Wang Z, Oron E, Nelson B, Razis S and Ivanova N: Distinct lineage specification roles for NANOG, OCT4, and SOX2 in human embryonic stem cells. Cell Stem Cell 10: 440-454, 2012.

29. Moon JH, Heo JS, Kim JS, et al: Reprogramming fibroblasts into induced pluripotent stem cells with Bmi1. Cell Res 21: 1305-1315, 2011. 